\title{
Precision Targeting of Liver Lesions with a Needle-Based Soft Tissue Navigation System
}

\author{
L. Maier-Hein ${ }^{1}$, F. Pianka ${ }^{2}$, A. Seitel ${ }^{1}$, S.A. Müller ${ }^{2}$, A. Tekbas ${ }^{2}$, M. Seitel $^{1}$, \\ I. Wolf ${ }^{1}$, B.M. Schmied ${ }^{2}$, and H.-P. Meinzer ${ }^{1}$ \\ ${ }^{1}$ German Cancer Research Center, Div. Medical and Biological Informatics, \\ Im Neuenheimer Feld 280, 69120 Heidelberg \\ 1.maier-hein@dkfz.de \\ ${ }^{2}$ University of Heidelberg, Dept. of General, Abdominal and Transplant Surgery \\ Im Neuenheimer Feld 110, 69120 Heidelberg, Germany ${ }^{\star}$
}

\begin{abstract}
In this study, we assessed the targeting precision of a previously reported needle-based soft tissue navigation system. For this purpose, we implanted $102-\mathrm{ml}$ agar nodules into three pig livers as tumor models, and two of the authors used the navigation system to target the center of gravity of each nodule. In order to obtain a realistic setting, we mounted the livers onto a respiratory liver motion simulator that models the human body. For each targeting procedure, we simulated the liver biopsy workflow, consisting of four steps: preparation, trajectory planning, registration, and navigation. The lesions were successfully hit in all 20 trials. The final distance between the applicator tip and the center of gravity of the lesion was determined from control computed tomography (CT) scans and was $3.5 \pm 1.1 \mathrm{~mm}$ on average. Robust targeting precision of this order of magnitude would significantly improve the clinical treatment standard for various CT-guided minimally invasive interventions in the liver.
\end{abstract}

\section{Introduction}

Computer tomography (CT) guided minimally invasive procedures in the liver such as tumor biopsy and thermal ablation therapy frequently require the targeting of hepatic structures that are subject to breathing motion. Unfortunately, commercially available navigation systems are still restricted to applications for rigid structures, such as the skull and the spine.

To allow application of existing navigation techniques to the liver, several research groups (e.g. 1 1234567 ) are investigating methods for compensating organ motion during soft tissue interventions; however, a common approach for assessing the accuracy of the navigation systems developed in this context has not yet been established. Zhang et al. 5] implanted tumor models containing radio-opaque $\mathrm{CT}$ contrast medium into a silicon liver model mounted on

\footnotetext{
* The present study was conducted within the setting of "Research training group 1126: Intelligent Surgery" funded by the German Research Foundation (DFG).
} 


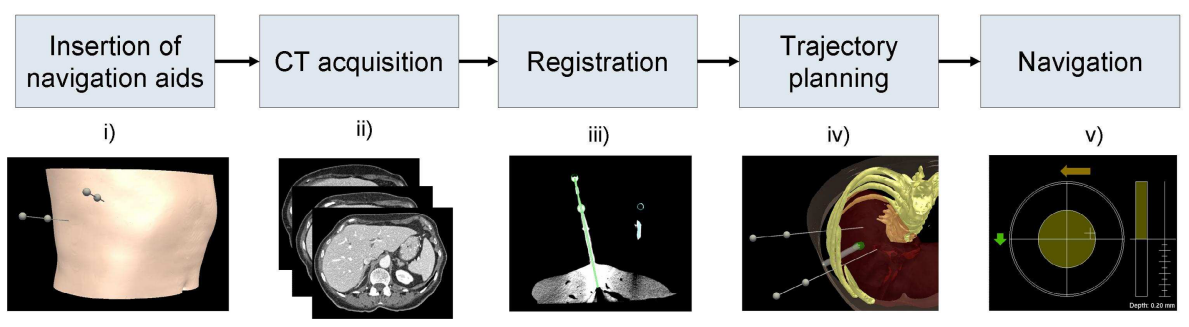

Fig. 1. Our soft tissue navigation concept. i) The navigation aids are inserted in the vicinity of the target. ii) A planning computed tomography (CT) scan is acquired. iii) The navigation aids are registered with the planning CT image, and the tracking coordinate system is registered with the CT coordinate system. iv) The navigation target point is chosen, and a trajectory is planned. v) A real-time deformation model is used to continuously estimate the position of the target point from the current positions of the optically tracked navigation aids, and a navigation display supports the targeting process accordingly.

a motion simulator and also conducted experiments in swine with agar injections as nodular targets. Kahn et al. 6] evaluated their navigation system in human cadavers, with three different targets: a predefined position within the ascending aorta, a calcified plaque in an artery, and the tip of a port catheter. Fichtinger et al. [8] conducted experiments in ventilated swine cadavers and used stainless-steel staples as targets. Several other studies were performed with rigid phantoms and did not incorporate organ shift or deformation (e.g. [34]).

In a previous report [7, we introduced a needle-based navigation system for minimally invasive interventions in the liver, in which a real-time deformation model is used to estimate the position of a navigation target point continuously from a set of optically tracked navigation aids (Fig. 1). The accuracy of tracking, CT registration, and target position estimation throughout the breathing cycle have already been evaluated [79. We have also investigated suitable visualization schemes to support soft tissue targeting procedures in cooperation with clinicians 10 .

In this study, we assessed the overall targeting accuracy of the system and present a general workflow for evaluating the performance of a liver navigation system in a realistic setting.

\section{Material and Methods}

Our approach for assessing the targeting precision of our liver navigation system is based on simulation of the clinical liver biopsy workflow for porcine livers mounted onto a respiratory motion simulator. We used injected agar nodules as tumor models and determined the targeting error from control CT scans. The following sections describe the workflow in detail and present the experimental conditions used in this study. 


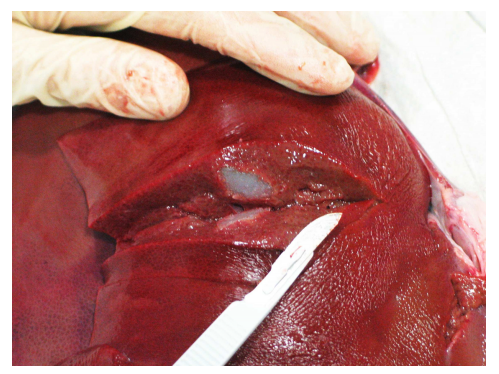

a)

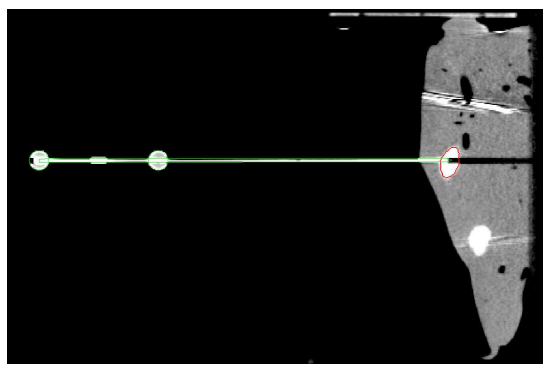

b)

Fig. 2. Agar nodule in a porcine liver (a) and in a control CT image (b)

\section{$2.1 \quad$ Workflow}

Each targeting procedure comprises four steps: preparation, trajectory planning, registration, and navigation, as well as a post-processing procedure. While the preparation step is conducted only once for each liver, the trajectory must be planned separately for each lesion, and the remaining steps have to be repeated for each trial. The evaluation procedure was designed specifically for our navigation system but could readily be adapted for other navigation methods.

The detailed workflow used for this study was as follows:

1. Preparation: We prepared each porcine liver according to the following procedure:

(a) Based on the method proposed by Zhang et al. [5], a 5\% agar dilution was prepared and mixed with contrast agent $(1: 15 \mathrm{v} / \mathrm{v}$ dilution).

(b) Three to four agar nodules of volume $2 \mathrm{ml}$ were then injected into the liver (Fig. 2a). In case of a spherical lesion, a volume of $2 \mathrm{ml}$ corresponds to a diameter of approximately $1.5 \mathrm{~cm}$.

(c) The liver was sewn to the diaphragm model (i.e., the Plexiglas ${ }^{\circledR}$ plate) of the motion simulator introduced in [9] (Fig. 33).

(d) Two 5 Degrees-of-Freedom (5DoF) navigation aids [7] were inserted into the liver ("diagonal arrangement", Fig. 4b).

(e) A planning CT scan of the motion simulator with the integrated porcine liver was acquired (Somatom Sensation 16 multidetector row scanner; Siemens, Erlangen, Germany). A fine resolution (0.75 $\mathrm{mm}$ slices) was necessary because our evaluation relies on accurate computation of the center of gravity of the agar nodule in both the planning CT and the control CT.

(f) The motion simulator was used to simulate several breathing cycles (cranio-caudal displacement of the liver $\approx 15 \mathrm{~mm}[9$ ) reflecting the fact that the patients cannot hold their breaths between acquisition of the planning $\mathrm{CT}$ and registration. 


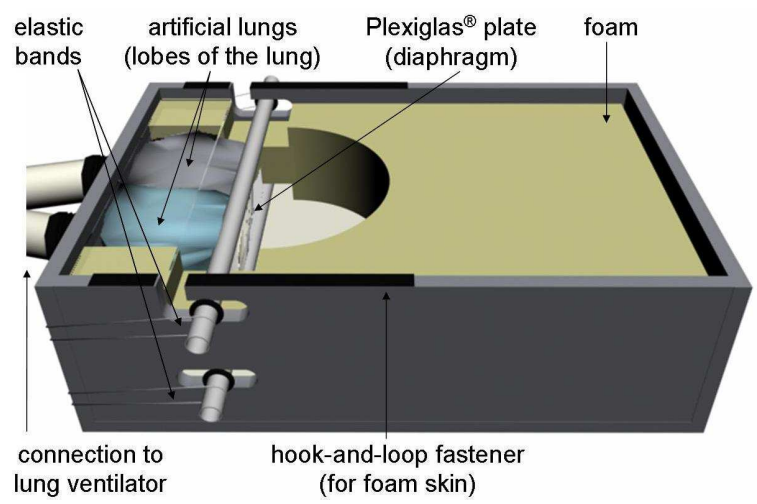

Fig. 3. Schematic view of the respiratory liver motion simulator

2. Trajectory planning: For each lesion, we planned a trajectory in the CT image as follows:

(a) The tumor was segmented semi-automatically on the basis of the graphcut algorithm [11.

(b) The navigation target point was set to the center of gravity of the segmented tumor.

(c) An insertion point was chosen on the skin.

3. Registration: On the basis of the planned trajectory, we performed the initial registration:

(a) The navigation aid models were registered with the planning CT image by the semi-automatic algorithm described in [9].

(b) The tracking coordinate system was registered with the CT coordinate system. For this purpose, we used the optical markers on the navigation aids as fiducials to compute a landmark-based rigid transformation as described in [7].

4. Navigation: We used an optically tracked applicator to target a given agar nodule with the navigation system. The targeting procedure was conducted at end-expiration because it represents the natural state of the motion simulator (with the artificial lungs relaxed). As we performed gated experiments and only two navigation aids were utilized for motion compensation we chose a rigid deformation model [9]. A navigation monitor provided the visualization for the targeting process:

(a) A two-dimensional projection view and a tool tip camera guided the user through the three steps tip positioning, needle alignment, and needle insertion as described in [10].

(b) Once the target was reached, the current position of the applicator was recorded. Then, the tool was released and its position was recorded again. The resulting tip "offset" was stored in image coordinates. This step was necessary because of the lack of tissue between the skin of the motion simulator (the foam) and the liver (Fig. 4b); once the applicator was released, the elastic skin relaxed and potentially pulled the tool several millimeters out of the liver. 


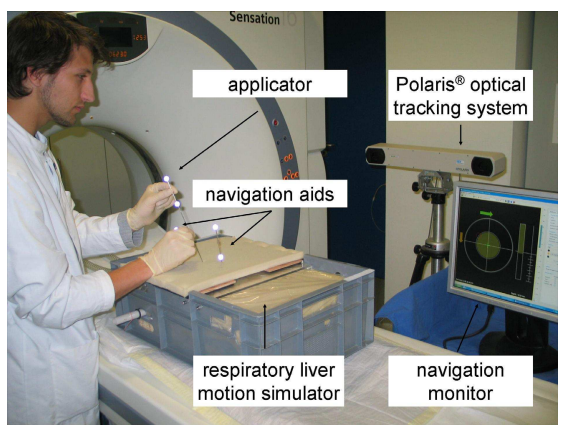

a)

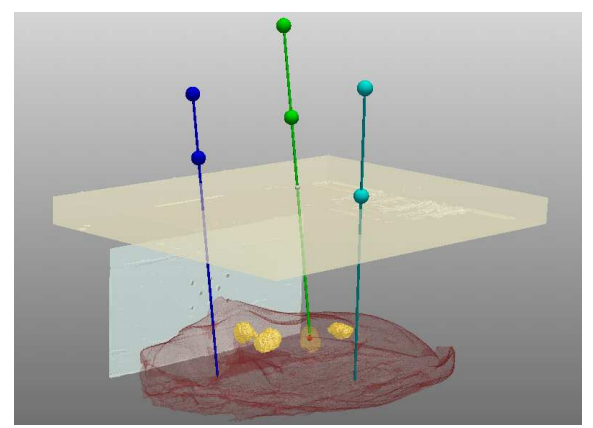

b)

Fig. 4. Navigation scenario: (a) Experimental setup for the targeting procedure, and (b) reconstructed three-dimensional view showing the liver (brown) with four injected agar nodules (yellow), the inserted applicator (green), the two navigation aids (blue and turquoise), the Plexiglas ${ }^{\circledR}$ plate as diaphragm model (light blue), the artificial skin (beige), the insertion point on the skin (white), and the target point (red)

5. Post-processing: The targeting accuracy was determined with a control CT:

(a) A CT scan was acquired with the same settings as for the planning CT.

(b) The tumor in the control CT image was segmented semi-automatically with the graph-cut algorithm 11 .

(c) The navigation target point was set to the center of gravity of the segmented tumor as reference.

(d) The applicator model was registered with the control CT image by the semi-automatic algorithm described in [7].

(e) The position of the applicator was corrected by the offset computed in the navigation step.

(f) The distance between the computed target point and the (corrected) position of the applicator tip was recorded as the CT targeting error $\epsilon^{C T}$.

\subsection{Experimental Conditions}

In order to determine the overall targeting error of our navigation system, one technician (S1) and one fourth-year medical student (S2) conducted 20 targeting procedures in 10 tumor lesions following the workflow described above. Each participant simulated one biopsy from each lesion (Fig. 4a), and we recorded the following errors:

- the fiducial registration error (FRE) which is the mean distance between the optical markers in image coordinates and the transformed optical markers originally located in tracking coordinates as described in 9.

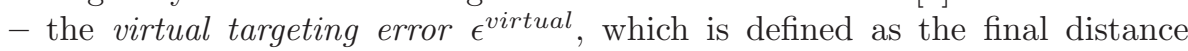
between the applicator tip (given by the tracking system) and the estimated target point position (according to the deformation model). This error results 
primarily from an inaccurate instrument insertion and depends crucially on the experience of the user.

- the CT targeting error $\epsilon^{C T}$ defined in section 2.1 (post-processing). It includes the registration error, the target position estimation error of the system, the tracking error, and the instrument insertion error. In addition, it is sensitive to changes in the applicator position between the instrument insertion step and the CT acquisition as discussed below.

\section{Results}

Our navigation system was successfully applied for simulating 20 liver biopsies according to the workflow described above. The applicator trajectory was generally non-parallel to the CT scanning plane, and the mean distance between the insertion point and the target point $( \pm \mathrm{SD})$ was $11.6 \pm 1.0 \mathrm{~cm}$.

Table 1. Virtual targeting error, $\epsilon^{\text {virtual }}$, and CT targeting error, $\epsilon^{C T}$, for participant $\mathrm{S} 1$, participant $\mathrm{S} 2$, and both participants $(\mathrm{S} 1, \mathrm{~S} 2)$ in $\mathrm{mm}$. The mean error $(\mu)$, the standard deviation $(\sigma)$, the root-mean-square $(R M S)$ error, the median error (median), and the maximum error $(\max )$ for the entire set of lesions are listed.

\begin{tabular}{l||c|c|c||c|c|c} 
& $\epsilon^{\text {virtual }} \mathbf{( S 1 )}$ & $\epsilon^{\text {virtual }} \mathbf{( S 2 )}$ & $\epsilon^{\text {virtual }}(\mathbf{S 1 , S 2})$ & $\epsilon^{C T} \mathbf{( S 1 )}$ & $\epsilon^{C T} \mathbf{( S 2 )}$ & $\epsilon^{C T} \mathbf{( S 1 , S 2 )}$ \\
\hline \hline$\mu \pm \sigma$ & $0.5 \pm 0.3$ & $1.1 \pm 1.1$ & $0.8 \pm 0.8$ & $\mathbf{2 . 8} \pm \mathbf{0 . 6}$ & $\mathbf{4 . 1} \pm \mathbf{1 . 1}$ & $\mathbf{3 . 5} \pm \mathbf{1 . 1}$ \\
$R M S$ & 0.6 & 1.5 & 1.1 & 2.9 & 4.3 & 3.6 \\
median & 0.4 & 0.7 & 0.6 & 3.0 & 4.2 & 3.3 \\
max & 1.3 & 4.0 & 4.0 & 3.8 & 5.4 & 5.4
\end{tabular}

The lesions were successfully hit in all trials with a mean fiducial registration error $( \pm \mathrm{SD})$ of $0.6 \pm 0.2 \mathrm{~mm}$ for computation of the coordinate transformation. The mean final distance $\epsilon_{\mu}^{C T}(S 1, S 2)$ between the applicator tip and the center of gravity of the segmented agar nodule was $3.5 \pm 1.1 \mathrm{~mm}$ averaged over all trials (Table 1).

If we regard the first trial of subject $\mathrm{S} 2$ an outlier $\left(\epsilon^{\text {virtual }}: 4.0 \mathrm{~mm}\right)$ and exclude it from consideration, the mean virtual targeting error was of the same order of magnitude for both participants $(<1 \mathrm{~mm})$. The mean CT targeting error was, however, significantly larger for S2 $(4.1 \pm 1.1 \mathrm{~mm})$ than for S1 $(2.8 \pm 0.6 \mathrm{~mm})$. In addition, the virtual targeting error $\epsilon^{\text {virtual }}$ estimated with our navigation system was generally significantly smaller than $\epsilon^{C T}$, averaging only $0.8 \pm 0.8 \mathrm{~mm}$.

\section{Discussion}

We assessed the targeting precision of a novel soft tissue navigation system and obtained a mean error of $3.5 \pm 1.1 \mathrm{~mm}$.

The proposed evaluation approach has three key features. First, we use agar nodules mixed with contrast agent as targets, as they are clearly distinguishable 
from the surrounding liver tissue and can thus be segmented easily. In addition, they can be prepared such that they resemble real tumors in terms of shape and size. A second key feature is the utilization of the motion simulator as body model allowing us to model organ movement due to respiration, the most challenging problem in soft tissue interventions. Finally, the evaluation is performed in-vitro allowing us to perform experiments in moving organs, without recourse to animal experiments, which are time-consuming and expensive. To our knowledge, we are the first to combine in-vitro experiments with simulation of respiratory motion.

The main drawback of our evaluation approach is the suboptimal fixation of the applicator in the body model. In our experience, small movements of the tool can occur relatively easily once it has been released, because it is held in position only by a layer of foam, several millimeters of (elastic) liver tissue and the relatively soft agar nodule itself (Fig. 4b). In other words, there is no assurance that the applicator will not shift further after the offset correction which potentially leads to inaccurate determination of the final applicator position and hence to an inaccurate error calculation. We consider that the large deviation between the virtual targeting error $\epsilon_{\mu}^{\text {virtual }}$ and the CT targeting error $\epsilon_{\mu}^{C T}$ can be attributed to this phenomenon. Similarly, we consider that the relatively large difference between the two observers with regard to $\epsilon_{\mu}^{C T}$ was due to inaccurate determination of the applicator tip offset. The technician (S1), who was more experienced in use of the system, released the applicator very carefully after each targeting and calculated the offset correction only after ensuring that the applicator had assumed its final position and showed no more movement. We assume that the other participant (S2) conducted the process less carefully, causing a less accurate offset computation. In order to overcome these limitations, we propose use of a real biopsy needle as the applicator and marking of the final tip position with injected material.

It is worth noting, that the navigation aids were better affixed within the tissue than the instrument because they were generally inserted considerably deeper into the liver (Fig. 4) and were less effected by the resilience of the foam. Since the same planning CT scan was used for all trials in one liver and the axes of the needles were nonparallel to each other, a shift of the navigation aids during one targeting procedure would have increased the registration error of the next trial. We obtained a very low FRE of only $0.6 \mathrm{~mm}$ on average, which suggests that the fixation of the navigations aids was sufficient. Moreover, the CT targeting error did not increase over time. To avoid problems related to this issue, however, we propose attaching the navigation aids to the skin.

Despite the technical problems discussed above, our accuracy is higher than that published in related work. Zhang et al. 5] reported a success rate of $87.5 \%(\mathrm{n}=16)$ in a silicon liver mounted on a motion simulator and a median targeting error of $8.3 \pm 3.7 \mathrm{~mm}(\mathrm{n}=32)$ in swine. Other groups obtained mean errors of $8.4 \pm 1.8 \mathrm{~mm}(\mathrm{n}=42)$ in human cadavers [6] and $6.4 \pm 1.8(\mathrm{n}=22)$ in ventilated swine [8].

We evaluated the targeting precision of our needle-based soft tissue navigation system in-vitro and obtained a mean error of $3.5 \pm 1.1 \mathrm{~mm}$. Our clinical 
colleagues have commented that a robust targeting precision of this order of magnitude would improve the treatment standard for CT-guided minimally invasive interventions in the liver dramatically. In order to advance clinical application of our navigation method, we are currently planning experiments in swine.

\section{References}

1. Schweikard, A., Glosser, G., Bodduluri, M., Murphy, M.J., Adler, J.R.: Robotic motion compensation for respiratory movement during radiosurgery. Comp. Aid. Surg. 5, 263-277 (2000)

2. Khamene, A., Warzelhan, J.K., Vogt, S., Elgort, D., Chefd'Hotel, C., Duerk, J.L., Lewin, J.S., Wacker, F.K., Sauer, F.: Characterization of internal organ motion using skin marker positions. In: Barillot, C., Haynor, D.R., Hellier, P. (eds.) MICCAI 2004. LNCS, vol. 3217, pp. 526-533. Springer, Heidelberg (2004)

3. Nagel, M., Schmidt, G., Petzold, R., Kalender, W.A.: A navigation system for minimally invasive CT-guided interventions. In: Duncan, J.S., Gerig, G. (eds.) MICCAI 2005. LNCS, vol. 3750, pp. 33-40. Springer, Heidelberg (2005)

4. Nicolau, S., Garcia, A., Pennec, X., Soler, L., Ayache, N.: An augmented reality system to guide radio-frequency tumour ablation. Comput. Animat. Virt. W 16, $1-10(2005)$

5. Zhang, H., Banovac, F., Lin, R., Glossop, N., Wood, B.J., Lindisch, D., Levy, E., Cleary, K.: Electromagnetic tracking for abdominal interventions in computer aided surgery. Comp. Aid. Surg. 11(3), 127-136 (2006)

6. Khan, M.F., Dogan, S., Maataoui, A., Wesarg, S., Gurung, J., Ackermann, H., Schiemann, M., Wimmer-Greinecker, G., Vogl, T.J.: Navigation-based needle puncture of a cadaver using a hybrid tracking navigational system. Invest. Radiol. 41(10), 713-720 (2006)

7. Maier-Hein, L., Maleike, D., Neuhaus, J., Franz, A., Wolf, I., Meinzer, H.P.: Soft tissue navigation using needle-shaped markers: Evaluation of navigation aid tracking accuracy and CT registration. In: SPIE Medical Imaging 2007: Visualization, Image-Guided Procedures, and Display, vol. 6509, p. 650926 (2007)

8. Fichtinger, G., Deguet, A., Fischer, G., Iordachita, I., Balogh, E., Masamune, K., Taylor, R.H., Fayad, L.M., de Oliveira, M., Zinreich, S.J.: Image overlay for CTguided needle insertions. Comp. Aid. Surg. 10(4), 241-255 (2005)

9. Maier-Hein, L., Müller, S.A., Pianka, F., Müller-Stich, B.P., Gutt, C.N., Seitel, A., Rietdorf, U., Meinzer, H.P., Richter, G., Schmied, B.M., Wolf, I.: In-vitro evaluation of a novel needle-based soft tissue navigation system with a respiratory liver motion simulator. In: SPIE Medical Imaging 2007: Visualization, Image-Guided Procedures, and Display, vol. 6509, p. 650916 (2007)

10. Seitel, A., Maier-Hein, L., Schawo, S., Radeleff, B.A., Mueller, S.A., Pianka, F., Schmied, B.M., Wolf, I., Meinzer, H.P.: In-vitro evaluation of different visualization approaches for computer assisted targeting in soft tissue. In: CARS. Computer Assisted Radiology and Surgery (to appear, 2007)

11. Boykov, Y., Kolmogorov, V.: An experimental comparison of min-cut/max-flow algorithms for energy minimization in vision. IEEE T Pattern Anal 26(9), 11241137 (2004) 